\title{
INVESTIGACIONES
}

\section{Evaluación en contexto MAPUCE BAFKEHCE: las voces del profesorado en la Araucanía*}

\author{
Evaluation in context MAPUCE BAFKEHCE: \\ the voices of teachers in Araucanía \\ Avaliação no contexto MAPUCE BAFKEHCE: \\ as vozes dos professores em Araucanía
}

\author{
Miguel Del Pino ${ }^{a}$, Froilán Cubillos ${ }^{b}$, Diego Pinto \\ ${ }^{a}$ Vicerrectoría de Investigación y Posgrado. Centro de Investigación en Educación para la Justicia Social, \\ CIEJUS, Universidad Católica del Maule, Chile. \\ mdelpino@ucm.cl \\ ${ }^{b}$ Centro de Investigación en Educación para la Justicia Social, \\ CIEJUS, Universidad Metropolitana de Ciencias de la Educación, Chile. \\ froycub@yahoo.es \\ ${ }^{c}$ Centro de Investigación en Educación para la Justicia Social, CIEJUS, \\ Universidad Metropolitana de Ciencias de la Educación, Chile. \\ dialpive@gmail.com
}

\begin{abstract}
RESUMEN
Esta investigación se realizó en contexto indígena MAPUCE BAFKEHCE, en la Región de la Araucanía, Chile. Con el fin de generar una evaluación de aprendizajes que sea pertinente a su cultura y lengua, se propuso como objetivos: 1) Identificar los principios que orientan la evaluación de aprendizajes en contexto MAPUCE BAFKEHCE junto a la comunidad y 2) Definir el concepto de evaluación de aprendizajes en contexto MAPUCE BAFKEHCE y su metodología. El estudio se abordó desde el paradigma participativo y el enfoque dialógicokishu kimkelay ta che, junto con profesorado de una escuela MAPUCE, por medio de conversaciones dialógicas y el análisis de fuentes documentales. Los resultados evidencian principios orientadores que promueven una evaluación situada territorial, cultural y lingüísticamente; como también, propuestas metodológicas coherentes a la cultura indígena. Con la finalidad de revitalizar sus saberes desde una óptica evaluativa que permita establecer un diálogo con la escolarización chilena occidental.
\end{abstract}

Palabras claves: Kimvn, lengua indígena, metodología de evaluación.

\section{ABSTRACT}

This research was conducted in the indigenous context MAPUCE BAFKEHCE, in the Araucanía Region, Chile. In order to generate a learning assessment that is relevant to their culture and language, the following objectives

\footnotetext{
Investigación financiada por el Proyecto Mejoramiento Institucional, PMI UMCE 1501. Universidad Metropolitana de Ciencias de la Educación (Santiago, Región Metropolitana). Código PMI-EXA-PNII-02-2017.

Para las palabras y conceptos de la cultura MAPUCE se utiliza el grafemario Raguileo, por respeto a la decisión propia de la comunidad con la que se desarrolló esta investigación. Así también, toda palabra en lengua MAPUCE se coloca en mayúscula, bajo el principio de respeto hacia la cultura originaria.
} 
were proposed: 1) Identify the principles that guide the evaluation of learning in the context of MAPUCE BAFKEHCE together with the community and 2) Define the concept of evaluation of learning in context MAPUCE BAFKEHCE and its methodology. The study was approached from the participative paradigm and the dialogical-kishu kimkelay ta che approach, together with the teachers of a MAPUCE school, through dialogical conversations and the analysis of documentary sources. The results show guiding principles that promote an evaluation located territorially, culturally and linguistically; as well as methodological proposals coherent to the indigenous culture. In order to revitalize their knowledge from an evaluative perspective that allows to establish a dialogue with western chilean schooling.

Key words: Kimvn, indigenous language, evaluation methodology.

\section{RESUMO}

Esta pesquisa foi realizada no contexto indígena MAPUCE BAFKEHCE, na Região de Araucanía, Chile. A fim de gerar uma avaliação de aprendizagem que seja relevante para sua cultura e linguagem. Ele propôs os seguintes objectivos: 1) Identificar os princípios que guiam a avaliação da aprendizagem no contexto mapuche BAFKEHCE com a comunidade e 2) definir o conceito de avaliação da aprendizagem no contexto mapuche BAFKEHCE e metodologia. O estudo foi abordado a partir do paradigma participativo e os kishu abordagem dialógicas kimkelay ta-che, juntamente com professores da escola mapuche, através de discussões e análise de fontes documentais dialógicas. Os resultados mostram princípios norteadores que promovem uma avaliação localizada territorial, cultural e linguisticamente; bem como propostas metodológicas coerentes com a cultura indígena. A fim de revitalizar seus conhecimentos a partir de uma perspectiva avaliativa que permita estabelecer um diálogo com a escolaridade chilena ocidental.

Palavras-chave: Kimvn, língua indígena, metodologia de avaliação.

\section{INTRODUCCIÓN}

En las últimas décadas cobra gran relevancia la crítica de los pueblos indígenas a nivel mundial respecto a la temática de evaluación educativa, manifestando entre otras cosas, que la evaluación se ha centrado en el logro de aprendizajes estandarizados y que todavía se concibe desde una concepción tradicional en su aplicación en el aula (Calvino, 2013; Del Pino, Pino y Manosalva, 2018). Es decir, la evaluación no tiene sentido de pertinencia cultural para escuelas ubicadas en contexto educativos con población indígena.

Se encuentra en la literatura científica la demanda por una evaluación de aprendizajes que sea pertinente al contexto educativo. Por un lado, existen antecedentes jurídicos que respaldan la importancia de desarrollar una educación que incluya saberes y la enseñanza de las distintas lenguas de comunidades indígenas del continente latinoamericano, un claro ejemplo de esto es la impronta de los Programas de Educación Intercultural (Williamson y Flores, 2015). A nivel mundial, la Declaración de las Naciones Unidas sobre los Derechos de los Pueblos Indígenas (Naciones Unidas, 2007), el Convenio 169 de la OIT sobre Pueblos Indígenas y Tribales (Organización Internacional del Trabajo, 1989), demuestran la preocupación acerca de mejorar las condiciones escolares y educativas de los distintos pueblos indígenas.

Por otro lado, encontramos antecedentes en la literatura científica acerca de evaluación educativa en contexto indígena. Mertens (2013), ha desarrollado el paradigma transformador en evaluación, que tiene como foco de atención los derechos humanos y la justicia social, dirigida a los grupos minoritarios como grupos étnicos, de género, de niños e inmigrantes. De esta manera, evaluar es prestar atención a la diversidad en un 
trabajo evaluativo en conjunto con las personas y comunidades. Similar a este trabajo, Cram $(2001 ; 2009)$ ha desarrollado la evaluación en contexto maorí en Nueva Zelanda, incorporando una praxis territorializada, siendo pertinente a las prácticas ancestrales y la lengua indígena. Ambas autoras plantean la necesidad de un paradigma transformador y participativo en evaluación en contexto indígena (Cram y Mertens, 2016). Paradigma que surge de la necesidad educativa de los propios pueblos indígenas, donde la variable "contexto situado" es importante, debido que da la particularidad de que la evaluación se resignifica, teniendo sentido de realidad para las personas y no solamente es entendida como un mecanismo regulador desde una racionalidad occidental.

En contexto latinoamericano, el trabajo desarrollado por el Instituto Nacional de Evaluación Educativa en México (INEE), ha avanzado en evaluación en contexto intercultural, con población indígena y no-indígena, planteando que las evaluaciones estandarizadas deben evitar la discriminación en contra de las poblaciones minoritarias, por lo que se debe desarrollar una evaluación en lengua indígena. Para ello, el INEE, desarrolló un protocolo para la aplicación de evaluaciones estandarizadas en contexto indígena, como también, un protocolo para el profesorado (Schmelkes, 2015).

En Chile, sobre la base de la Ley indígena 19.253 (1993) y a partir del año 1996, se instala el Programa Educativo Intercultural Bilingüe (PEIB), que según el Ministerio de Educación (Mineduc) es un programa que busca contribuir al desarrollo de la lengua y cultura de los pueblos originarios en el territorio nacional y a la formación de ciudadanos interculturales en el sistema educativo. El PEIB está dirigido a sectores educativos que concentran mayor cantidad de estudiantes indígenas, sobre todo de sectores rurales (Chile Decreto $\mathrm{N}^{\circ}$ 280, 2009). Priorizando la enseñanza de las distintas lenguas originarias en este tipo de estudiantado, por medio de los programas curriculares del subsector de lengua indígena. Pero el mismo decreto hace una distinción que implica un carácter diferenciado para la obligatoriedad del sector de lengua indígena, considerando que los establecimientos educativos con alta densidad de población escolar indígena deben ofrecer esta materia (optativa para el alumno) y para el resto de las escuelas del país, tiene carácter de recomendación y está orientada a favorecer el bilingüismo y la interculturalidad (Lagos, 2015). Debido a lo anterior, el programa solo se ha implementado en escuelas con alta tasa de estudiantes indígenas, y han incorporado al aula educadores tradicionales, que son personas que pertenecen a un pueblo indígena y actúan como un apoyo pedagógico para el docente (Acuña, 2012).

En el contexto chileno, si bien existen avances en la inclusión de algunas temáticas educativas de los pueblos originarios, no ha sido en acuerdo con los propios movimientos indígenas, y una problemática que surge es el poco avance en materia de evaluación de aprendizajes para contextos escolares con población indígena y no indígena. Los programas curriculares de lengua indígena, no presentan indicadores pertinentes a la cultura de los pueblos originarios, entendiéndose la evaluación en términos occidentales. Este tipo de estudiantado se ve obligado a rendir las evaluaciones estandarizadas nacionales para su medición y continuación exitosa en el sistema educativo. En este sentido, una de las críticas que mayor fuerza cobra en nuestros países es que los estados insisten en utilizar pruebas estandarizadas para evaluar los aprendizajes, pruebas que no están adaptadas a la diversidad cultural, que entienden a toda la población estudiantil homogénea. La evaluación educativa no está definida por criterios de pertinencia cultural y menos orientada a satisfacer las necesidades particulares de los pueblos indígenas (Yopo, 2012). 
En este escenario, la presente investigación se sitúa en la Región de la Araucanía, Chile, lugar donde cohabita el pueblo MAPUCE, que según el territorio donde viven, tienen distintas denominaciones. En la Araucanía se han situado los PICUNCHE, gente del norte; NAGCHE, gente que vive en las tierras bajas (valle central); WENTECHE, gente que habita las planicies altas y BAFKEHCE, gente que habita la zona costera. Específicamente, se trabajó junto a la comunidad MAPUCE BAFKEHCE.

Esta comunidad indígena ha rechazado directamente el PEIB porque lo comprenden como un mecanismo de opresión impuesto desde el Estado; por lo que han reorientado su accionar hacia una propuesta educativa que les permita mantener su cultura y lengua al mismo tiempo que interactuar con la cultura occidental, pero esta vez desde la perspectiva de las propias comunidades indígenas. Así, esta investigación se circunscribe a una experiencia pedagógica que ha sido desarrollada en los últimos doce años (desde el año 2006). La zona geográfica donde se ubica esta comunidad indígena es Llaguepulli (JAQEPVJV), península lacustre en la ribera del Lago Budi (el lago salado más austral del mundo), perteneciente a la comuna de Teodoro Schmidt.

La escuela donde se realizó la investigación perteneció más de treinta años a una congregación religiosa, hasta que dicha congregación realizó un despido masivo de los y las docentes que habitaban en el territorio. Sumado a lo anterior, el territorio donde se ubica la escuela pertenece al padre de una PAPAY del sector (anciana-sabia), y cuando hubo el despido masivo de docentes, las autoridades MAPUCE decidieron comenzar la recuperación de sus tierras y de la escuela. Es así como, a partir del año 2006 se concreta el traspaso de la escuela a la comunidad, conformando la Sociedad Educacional KIMENTUCHEFE (Rivera y Fuentes, 2017).

Desde la recuperación de la escuela, las autoridades MAPUCE junto al profesorado y el LOF (comunidad), deciden comenzar una construcción curricular, amparándose en el Decreto 40 (Mineduc, 1996) que otorga la posibilidad de crear planes y programas propios a las instituciones educativas. Esta decisión tiene como fundamento el rechazo del Programa de Educación Intercultural emanado desde el Ministerio de Educación chileno, debido que la comunidad lo considera hegemónico.

Así, dentro de las actuales propuestas de educación intercultural en Chile existen grandes desacuerdos respecto a las formas en que se integran diversas culturas, en nuestro caso en particular, las culturas chilena occidental y MAPUCE BAFKEHCE. Como la relación de las culturas es desigual, tiende el proceso educativo a imponer la cultura occidental sobre la cultura MAPUCE, a través de una relación hegemónica y extreyendo solo aquellos conocimientos MAPUCE integrables y abordables con las formas de conocer y enseñar el mundo de la cultura occidental (Fernández et al., 2012, p. 7).

Es en este contexto educativo donde la comunidad MAPUCE BAFKEHCE invita a académicos universitarios, estudiantes de pregrado y posgrado a colaborar, junto con profesores y profesoras de la escuela, autoridades del LOF, ancianos y familias, a sistematizar sus conocimientos (KIMVN) y pensamiento (RAKIZVAM) para organizarlos curricular y pedagógicamente en programas de estudio propios.

El contexto escolar en el que se encuentra la escuela motivó a la comunidad MAPUCE BAFKEHCE a situar la problemática en evaluacion de aprendizajes, por lo que se plantearon preguntas orientadoras: ¿Cuáles son los principios que orientan una evaluación de aprendizajes 
para el contexto indígena que tengan pertinencia social, cultural, territorial y linguística?, ¿qué concepto de evaluación reúne los elementos propios de la comunidad indígena y promueve una metodología coherente a sus prácticas ancestrales? Estas problemáticas son las que abarca el presente estudio a través de los siguientes objetivos de investigación:

1) Identificar los principios que orientan la evaluación de aprendizajes en contexto MAPUCE BAFKEHCE junto a la comunidad.

2) Definir el concepto de evaluación de aprendizajes en contexto MAPUCE BAFKEHCE y su metodología.

\section{MARCO TEÓRICO}

En evaluación de aprendizajes en contexto indígena, existen avances en las últimas décadas que dan cuenta que la evaluación debe estar al servicio y ser herramienta de valoración de un contexto situado, comprendiendo las nociones de tiempo y espacio, los valores comunitarios, la historia, el territorio y la lengua indígena (Calvino 2013; Cram y Mertens, 2016; Schmelkes, 2015; Kawakami, Aton, Cram, Lai y Porima, 2007).

Con relación a lo anterior, la propuesta de Cram y Mertens (2016) "represents a worldview based on the premise that researchers and evaluators have an ethical responsibility to address issues of social justice and human rights." (p. 163). En este sentido, el trabajo apunta a la negociación entre una cosmovisión transformadora de la realidad, desde una racionalidad occidental, con la cosmovisión indígena, planteando la necesidad de una negociación solidaria entre ambas culturas.

En consecuencia, los avances en evaluación en contexto indígena contemplan, a lo menos, tres dimensiones: a) evaluar en la lengua propia de la comunidad indígena, b) la participación conjunta de la comunidad en la toma de decisión evaluativa y c) la oralidad como método de evaluación.

a) En cuanto a este punto, se destaca la necesidad de "evaluar en la lengua propia, así como respecto de los aprendizajes que responden a las características culturales de los diversos grupos indígenas" (Schmelkes 2015, p. 62). Esto apunta básicamente al rol del profesorado en contexto indígena, considerando que la naturaleza ética del contexto puede comprenderse únicamente desde la lengua indígena. La importancia del manejo de la lengua indígena en el profesorado radica en que,

...conocemos el papel de la lengua en la valoración y el fortalecimiento de la cultura, por lo que este hecho es un primer paso en asegurar un enfoque intercultural en la evaluación, en este caso de los docentes indígenas (Schmelkes, 2015, p. 63).

El conocimiento de la lengua promueve la revitalización de los saberes propios de la cultura, como las diversas prácticas sociales indígenas. De esta manera, por ejemplo, para el pueblo MAPUCE es la lengua el medio por el cual se transmiten su conocimiento (KIMVN) y pensamiento (RAKIZVAM) con relación al territorio donde viven (Fernández et al., 2012). El conocimiento de la lengua promueve una evaluación con base en el componente axiológico que tiene que ver con el contexto socio-histórico, los derechos humanos, la justicia social y la disminución de las desigualdades en evaluación educativa, 
cuestión que se relaciona con la ubicación geográfica y territorial de los grupos indígenas, y para su comprensión y evaluación es necesario el conocimiento de su lengua.

b) La participación conjunta de la comunidad en la toma de decisión evaluativa, se basa en un trabajo productivo y organizado con base en el colectivo, teniendo como marco fundacional el reconocimiento de la herencia cultural de los pueblos indígenas (Mejía, 2014). Desde el punto de vista epistemológico, la experiencia en pedagogía popular con comunidades indígenas, visibilizan y organizan educativamente un saber y conocimiento no "sobre" esas culturas sino "desde y con" ellas.

Respecto a esto, la evaluación debe basarse en normas y criterios culturalmente apropiados, por esto es necesaria la construcción conjunta del marco normativo de la evaluación, no solo desde el profesorado, sino en coherencia con las propias prácticas colectivas ceremoniales indígenas, con la participación de los padres, ancianos y docentes, la participación de la propia comunidad y escuelas (King y Schielmann, 2004). En términos de estas autoras:

La evaluación y apreciación, entendidas como un proceso continuo y cooperativo en el aprendizaje y la enseñanza, no se limitan a comprobar el aprovechamiento escolar de los alumnos al final de un programa o de los periodos escolares establecidos, sino que comprenden diversas estrategias tales como observaciones, entrevistas formales, autoevaluaciones y evaluaciones continuas del aprovechamiento escolar a lo largo del programa o periodo escolar. Esto supone la participación de los siguientes protagonistas:

- Los ancianos y la comunidad;

- Los padres;

- Los docentes;

- Los educandos;

- Los consejos escolares y las autoridades de la educación; y

- Los investigadores (King y Schielmann, 2004, pp. 54-55).

La evaluación construye estrategias pertinentes y reconocidas por la comunidad indígena, que participan en ellas y contribuyen al aumento de niños, niñas y jóvenes arraigados sólidamente en sus culturas, su lengua, su cosmovisión, "con una buena formación cultural y capacitados para participar en entornos culturales distintos y lograr éxitos personales y académicos importantes y mensurables, inclusive en función de las normas de educación establecidas en el plano nacional" (p. 55).

c) En coherencia con las dimensiones anteriores, el método de evaluación que mayoritariamente se promueve desde las comunidades indígenas es la oralidad por medio de la narración (Calvino, 2013; Drawson, Toombs y Mushquash, 2017; Kawakami et al., 2007).

La narración, como método conversacional tiene mucha relación con las técnicas conversacionales occidentales. En la escuela occidental, se evalúa también de manera oral, tanto en exposiciones como en la autoevaluación. En contexto indígena la narración oral persigue:

... a connection to indigenous knowledge, a location within an Indigenous paradigm, a ralational natura, a purpose (wich is often decolonizing), following a specific protocol that reflects the Indigenous knowledge, a flexible natura, collaboration, and reflexivity (Kovach, 2010, citado en Drawson, Toombs y Mushquash, 2017, p. 4). 
Así como para la población occidental los recursos tecnológicos nos permiten guardar información relevante para preservarla, las comunidades indígenas (en su mayoría) tienen la memoria como soporte fundamental para el mantenimiento de su cultura (Quilaqueo y Quintriqueo, 2017). La transmisión de su cultura es oral, por esto la narración constituye una fuente de conocimiento y de aprendizaje. Explican los mismos autores que,

El discurso mapuche, con sus reglas, sus tipologías y la variabilidad de contenido, muestra no sólo una orientación hacia una oralidad afirmada a través de los tiempos, sino también, su utilización precisa en la organización de la vida social y educativa (Quilaqueo y Quintriqueo, 2017). Esto implica una tradición centenaria existente acerca de la palabra mapuche y del mantenimiento de hábitos sociales, lo que le ha dado cohesión como pueblo (p. 161).

La oralidad como método de evaluación introduce una concepción lingüística particularmente diferente que, en la escuela occidental, permitiendo una narración como proceso formativo no solo de un aprendizaje instrumental, sino de un discurso sociohistórico que da cuenta del aprendizaje en términos de transmisión cultural (Calvino, 2013). Este método se desarrolla en ambientes de cercanía, y se genera en dinámicas grupales de intercambio de saberes, enseñanza, evaluación, entre otros.

\section{METODOLOGÍA}

La investigación se circunscribe al paradigma participativo (Fals Borda, 1979; Heron y Reason, 1997), que permite una comprensión de la naturaleza de la realidad (ontología) desde una doble racionalidad cuando se trabaja junto a comunidades indígenas. Debido que, por un lado, la comunidad indígena convive con la realidad objetiva "colonial" occidental, y a la vez es una realidad subjetiva, que guarda relación con sus propias prácticas ancestrales, su lengua y cosmovisión. Así también, se comprende una epistemología extendida, que no se cierra a una única opción de formas de conocimiento, sino a una apertura epistémica.

En correspondencia con lo anterior, se trabajó con el enfoque de investigación dialógico-kishu kimkelay ta che (Ferrada et al., 2014; Ferrada, 2017; Ferrada y Del Pino, 2017). Dialógico, porque los problemas a investigar y la construcción de conocimientos se realizan y desarrollan junto a las personas situadas en sus contextos específicos. Y kishu kimkelay ta che, que en lengua MAPUCE significa que "ninguna persona conoce y/o aprende por si misma" sino en base a su legado histórico junto a las demás personas (Ferrada et al., 2014, p. 35).

Coherente al enfoque de investigación, se conformó una comunidad de investigación, compuesta por profesorado mapuce ( 2 hombres y 3 mujeres), 2 académicos de la ciudad de Santiago y 1 académico de la ciudad de Temuco. Esta comunidad de investigación decidió el problema a investigar que da cuenta situadamente de sus requerimientos en evaluación de aprendizajes.

Una vez constituida la comunidad de investigación, se decidió trabajar con los procedimientos de construcción de conocimiento propios del enfoque escogido: la conversación dialógica y la interpretación del discurso dialógico. La primera, permite una conversación en profundidad con personas que guardan un saber experiencial específico 
relacionado con la temática de investigación, en este caso con profesorado de la escuela. Y el segundo procedimiento, permite un análisis e interpretación de discursos, tanto orales como escritos, que se realiza juntamente con las personas de la comunidad de investigación, respecto del tema de estudio. Este procedimiento se utilizó con fuentes documentales, 2 tesis de pregrado y 2 capítulo de libro que recogen la experiencia pedagógica; todos textos desarrollados en la misma escuela en estos últimos años (2012, 2017 y 2018). Posteriormente, se transcribe toda la información recogida, realizándose un tratamiento de los datos y su categorización en dimensiones transformadoras y exclusoras. Las primeras dan cuenta de aquel conocimiento en evaluación de aprendizajes que la comunidad de investigación considera un aporte al proceso de mejora en la escuela, y la segunda, da cuenta de aquel conocimiento acerca de evaluación de aprendizajes que la misma comunidad considera que obstaculiza los procesos de mejora educativa que persiguen.

Una síntesis de la comunidad de investigación y de los procedimientos utilizados se detalla en la Tabla 1.

Tabla 1. Participantes de la investigación y procedimientos

\begin{tabular}{|l|l|}
\hline Comunidad de investigación & $\begin{array}{l}3 \text { profesoras MAPUCE + 2 profesores MAPUCE + 3 académi- } \\
\text { cos no indígenas. Total 8 personas }\end{array}$ \\
\hline $\begin{array}{l}\text { Procedimientos de construcción } \\
\text { de conocimiento }\end{array}$ & Conversación dialógica + interpretación del discurso dialógico \\
\hline Documentos analizados & $\begin{array}{l}\text { 2 tesis de pregrado (Fernández, Jofré, Mancini, Navarrete y Ve- } \\
\text { negas, 2012; Rivera y Fuentes, 2017) } \\
\text { 2 capítulos de libro (Calfuqueo, Cubillos, Pinto y Del Pino, } \\
\text { 2018; Del Pino, Pino y Manosalva, 2018) }\end{array}$ \\
\hline Tratamientos de datos & $\begin{array}{l}\text { Categorización de la información en dimensiones transforma- } \\
\text { doras y exclusoras. }\end{array}$ \\
\hline
\end{tabular}

Fuente. Elaboración propia.

\section{RESULTADOS}

En términos generales, los resultados permiten identificar dos grandes categorías específicas de la evaluación de aprendizajes en contexto mapuce. La primera categoría da cuenta de conceptos que orientan la evaluación, y la segunda categoría presenta el concepto de evaluación y sus distintos componentes según el contexto socio-histórico, cultural y lingüístico. Se presentan los resultados según las dos categorías mencionadas.

\subsection{RESULTADOS DE LA CATEGORÍA PRINCIPIOS QUE ORIENTAN LA EVALUACIÓN EN CONTEXTO MAPUCE}

Desde una comprensión general, los resultados del objetivo 1, Identificar los principios que orientan la evaluación de aprendizajes en contexto MAPUCE BAFKEHCE junto a la 
comunidad, son contenidos que ayudan a entender la evaluación desde una cosmovisión indígena, que difiere completamente de una racionalidad occidental. Estos conceptos son: KIMKANTUN (aprendizaje), MAPUZUGUN (lengua MAPUCE), KIMCE (sabio), KIMVN (conocimiento) y LOF (comunidad), como se observa en la Tabla 2.

Tabla 2. Principios que orientan la evaluación de aprendizajes en contexto MAPUCE

\begin{tabular}{|c|c|c|}
\hline Categorías & Conceptos & Contenidos de los conceptos \\
\hline \multirow{5}{*}{$\begin{array}{l}\text { Principios que orientan } \\
\text { la evaluación: principios } \\
\text { identitarios }\end{array}$} & $\begin{array}{l}\text { KIMKANTUN } \\
\text { (aprendizaje) }\end{array}$ & $\begin{array}{l}\text { 1. Aprender a conocer haciendo } \\
\text { 2. Conocer a partir de la practica }\end{array}$ \\
\hline & $\begin{array}{l}\text { MAPUZUGUN } \\
\text { (lengua mapuce) }\end{array}$ & $\begin{array}{l}\text { 1. Muestra de que su cultura sigue viva } \\
\text { 2. Ámbito del saber }\end{array}$ \\
\hline & $\begin{array}{l}\text { KIMCE } \\
\text { (sabio) }\end{array}$ & $\begin{array}{l}\text { 1. Persona sabia } \\
\text { 2. Etapa culmine del proceso de aprendizaje } \\
\text { 3. Saber ancestral }\end{array}$ \\
\hline & $\begin{array}{l}\text { KIMVN } \\
\text { (conocimiento) }\end{array}$ & $\begin{array}{l}\text { 1. Conocimientos } \\
\text { 2. Saberes }\end{array}$ \\
\hline & $\begin{array}{l}\text { LOF } \\
\text { (comunidad) }\end{array}$ & 1. Lazo afectivo \\
\hline
\end{tabular}

Fuente. Elaboración propia.

Desde una comprensión específica de cada concepto, la comunidad de investigación decidió categorizar todos en la dimensión transformadora, debido que permiten una comprensión epistémica diferente de la evaluación occidental. Para la escuela en contexto chileno, la evaluación se entiende como la medición (cuantificación, calificación) o la valoración (cualitativo) del logro de resultados de aprendizajes que pueden ser observables tanto en instrumentos objetivos (por medio de reactivos) o procesuales (por ejemplo, rúbricas, pautas de observación, entre otros). No obstante, los conceptos que propone el profesorado mapuce orientan una acción de la evaluación en función del "contexto situado". Es decir, la evaluación de aprendizajes debe dar respuesta a requerimientos y demandas de aprendizajes propias del contexto indígena, respetando sus principios identitarios como la lengua, sus sabios, su comunidad y territorio inmediato.

Con base en lo anterior, es que cada concepto promueve una acción evaluadora con sentido de valoración y respeto hacia su propia cultura sin desmedro de la cultura occidental.

KIMKANTUN (aprendizaje): este concepto lo define la comunidad como "aprender haciendo o participando de la cultura MAPUCE BAFKEHCE" (Rivera y Fuentes, 2017, p. 13). Así, este concepto se refiere básicamente que los niños, niñas y jóvenes MAPUCE conozcan las principales actividades productivas y sociales de su familia y comunidad. En evaluación, se traduce como aquel saber acerca de las prácticas propias de aprendizaje de su cultura y que deben ser valoradas coherentemente a su cultura y lengua. El aprendizaje no se evalúa en momentos estáticos del proceso de enseñanza, sino que se evalúa continuamente, por lo que KIMKANTUN da cuenta que el aprendizaje es cíclico y en 
constante movimiento. Por lo tanto, los procesos de enseñanza, aprendizaje y evaluación no son separados sino conjuntos. Como explica un profesor:

En la cultura nuestra, si bien no está explicitado o demostrado (la evaluación), sin embargo, están las evaluaciones de cómo los niños van aprendiendo o como se van desenvolviendo y lo aplica en la vida adulta (Extracto de conversación dialógica, profesor mapuce).

La cita explica que la evaluación y el KIMKANTUN tienen relación no sólo con los contenidos escolares, sino con aprendizajes para el desempeño de los niños en la sociedad, en la cultura propia y en la cultura occidental.

MAPUZUGUN (lengua mapuce): toda lengua guarda los conocimientos propios a las culturas. Así, el castellano (lengua oficial de Chile) permite la comunicación entre las personas y la transmisión de conocimientos de manera oral y escrita; la lengua MAPUCE permite el diálogo con el universo entero, es decir, con el mar, con las estrellas, con las montañas, con el cielo, y además entre las personas. Y transmite conocimientos, prácticas ancestrales como el desarrollo continuo de las mismas según los cambios en la sociedad occidental con la que la comunidad MAPUCE convive.

Por lo tanto, evaluar debe ser una acción en lengua propia, porque solo en MAPUZUGUN se accede a conocimientos, valores, prácticas socioculturales que permiten un diálogo coherente con el mundo del que los MAPUCE son parte. A diferencia de la evaluación en términos occidentales chilenos, donde el castellano permite evidenciar tanto oral como por escrito, el logro o no de un determinado aprendizaje instrumental.

Como dice una profesora, con relación al aprendizaje de los niños en la escuela que debe ser en su lengua propia:

... si el niño hace el intento por aprender, si intenta utilizar palabras en su lenguaje, si es capaz de hablar, esas son las cosas que se van evaluando (Extracto de Conversación dialógica, profesora mapuce).

Las lenguas originarias indígenas son el único acceso a una construcción epistémica evaluativa distinta y pertinente a sus requerimientos y demandas educativas.

$K I M C E$ (sabio): este concepto significa que el KIMCE es una persona adulta sabia, que guarda un saber único acerca de un determinado tipo de práctica ancestral o conocimiento acerca de la lengua, los alimentos, las plantas, el cultivo, etc. Así también, la categoría de KIMCE se da cuando una persona ( $c e$ ) culmina un proceso de aprendizaje, proceso que transcurre durante toda la vida.

En evaluación de aprendizajes, KIMCE es una categoría que promueve la adquisición y desarrollo del conocimiento en distintos ámbitos socioculturales, tanto domésticos como laborales. Dado que la comunidad de investigación distingue esta categoría como un eje orientador de la evaluación porque toda persona mapuce debe valorar sus conocimientos, como también, a las personas portadoras de estos conocimientos. Como explican Rivera y Fuentes (2017):

...el manejo del conocimiento no está restringido al rol docente, es más, dentro de esta realidad (MAPUCE) el profesor se presenta como un mediador o facilitador del proceso en el cual los protagonistas son los niños y niñas. El o la estudiante abandona la lógica de 
receptor de aprendizajes o mero acumulador, para transformarse en agente activo en su propia formación en la multiplicidad de dimensiones propuestas por la comunidad, en este sentido, dentro de esta concatenación de procesos de enseñanza/aprendizaje (p. 17-18).

Traducido a evaluación de aprendizajes, el KIMCE es una categoría que explica que la transmisión de conocimientos no es únicamente realizada por personas consideradas "expertas" (como se entiende el rol docente occidental) sino más bien por personas que portan un saber con base a su legado histórico y experiencial.

KIMVN (conocimiento): este concepto se define como conocimientos y saberes propios de la cultura mapuce. Cada práctica ancestral o cotidiana guarda un KIMVN, como también, el territorio inmediato donde viven las comunidades indígenas y el legado histórico que la cultura guarda y promueve. Es así, por ejemplo, que Fernández et al. (2012) explican que el lugar físico donde habita la comunidad mapuce de esta investigación provee de KIMVN y RAKIZVAM (pensamiento).

La cultura MAPUCE ha ido elaborando y transmitiendo un tipo de conocimiento que escapa a la lógica del pensamiento occidental y se desarrolla con relación al contacto con su territorio inmediato, es decir, el BEWFV FVZV (Lago Budi), la Cordillera de la costa y el BAFKEH (mar) desde donde proviene su KIMVN (conocimiento) y se desprende su RAKIZVAM (pensamiento). De este modo es imposible concebir a la cultura MAPUCE BAFKEHCE ajena a su territorio (pp. 4-5).

La evaluación de aprendizajes debe concebirse entonces como promotora de un KIMVN por medio del aprendizaje y valoración de todos los elementos que componen el territorio físico y espiritual donde vive la comunidad MAPUCE, y no solo centrarse en conocimientos prescritos por el currículum oficial.

LOF (comunidad): esta categoría se define como:

...entidad territorial constituida por grupos de familias extensivas, y cuya autoridad es el logko. El lof es un espacio territorial que no abarca solamente a la gente que vive en él, sino también a todos los elementos que lo componen (animales, plantas, seres espirituales, cerros, esteros, etc.) (Calfuqueo et al., 2018, p. 76).

En coherencia con los conceptos anteriores, LOF sintetiza la importancia del aprendizaje, la lengua, los sabios y/o ancianos, el conocimiento y pensamiento MAPUCE reunidos y desarrollados en la comunidad y territorio inmediato de los niños, niñas y jóvenes. El conocimiento paulatino del LOF permite en evaluación de aprendizajes el desarrollo continuado de la valoración y aprendizaje de los distintos componentes, tanto físico como espirituales que componen el territorio en el que vive la comunidad.

En síntesis, el objetivo 1, Identificar los conceptos que orientan la evaluación de aprendizajes en contexto MAPUCE BAFKEHCE junto a la comunidad, devela principios identitarios de la comunidad indígena que permiten una comprensión de la cosmovisión MAPUCE desde una episteme que enriquece y resignifica la evaluación de aprendizajes. De esta manera, se entiende por evaluación no solo una recogida de información para formular juicios de valor, que es como tradicionalmente se define, sino más bien, ampliando la noción anterior; evaluar es un dispositivo tanto epistémico como metodológico que promueve una 
comprensión del "contexto situado" de cualquier territorio donde se desarrolle. Dicho contexto connota de significado y finalidad común, según los distintos requerimientos y demandas que las comunidades indígenas persiguen.

\subsection{RESULTADOS DE LA CATEGORÍA CONCEPTO DE EVALUACIÓN EN CONTEXTO MAPUCE Y SU METODOLOGÍA}

Desde una comprensión general, los resultados del objetivo 2, Definir el concepto de evaluación de aprendizajes en contexto MAPUCE BAFKEHCE y su metodología, devela dos categorías. La primera, da cuenta del concepto de evaluación del aprendizaje para la comunidad MAPUCE, distinguiendo dos sub-categorías: Evaluación en coherencia a la identidad mapuce y Evaluación como proceso homogéneo. La segunda categoría da cuenta de los elementos metodológicos tales como las Estrategias e Instrumentos de evaluación, presentando dos sub-categorías: Observación del proceso educativo y la Escala numérica como instrumento. Coherente al enfoque de investigación dialógico asumido, cada resultado se categorizó en las dimensiones transformadoras y exclusoras según la validación realizada con la comunidad de investigación. Una síntesis de estos resultados se presenta en la Tabla 3.

Tabla 3. Concepto de evaluación en contexto MAPUCE y metodología.

\begin{tabular}{|l|l|l|l|}
\hline Categoría & \multicolumn{1}{|c|}{ Sub-categoría } & Elementos de la categoría & \multicolumn{1}{c|}{ Dimensión } \\
\hline $\begin{array}{l}\text { Evaluación de } \\
\text { aprendizaje }\end{array}$ & $\begin{array}{l}\text { Evaluación en coherencia a } \\
\text { la identidad mapuce }\end{array}$ & $\begin{array}{l}\text { Desde y en lo cotidiano } \\
\text { Continuamente dialogado }\end{array}$ & Transformadora \\
\cline { 2 - 5 } & $\begin{array}{l}\text { Evaluación como proceso } \\
\text { homogéneo }\end{array}$ & $\begin{array}{l}\text { Evaluación estandarizada } \\
\text { Responder a resultados para } \\
\text { el Mineduc }\end{array}$ & Exclusora \\
\hline $\begin{array}{l}\text { Estrategias e } \\
\text { Instrumentos de } \\
\text { evaluación }\end{array}$ & Observación y conversación & $\begin{array}{l}\text { Los/as estudiantes aprenden } \\
\text { en todo lugar y ocasión } \\
\text { Se les evalúa observando a } \\
\text { partir de los principios } \\
\text { identitarios }\end{array}$ & Transformadora \\
\cline { 2 - 5 } & Escala numérica & $\begin{array}{l}\text { Calificación acorde al con- } \\
\text { texto } \\
\text { Escalas numéricas represen- } \\
\text { tativas de 1 a 4, 8 o 12. } \\
\text { Concepto de Dualidad }\end{array}$ & Transformadora \\
\hline
\end{tabular}

Fuente. Elaboración propia.

\subsubsection{Resultados dimensión transformadora}

Los resultados que la comunidad de investigación categorizó como transformadores, son aquellos que promueven una evaluación de aprendizajes coherente a su contexto histórico, social, cultural y lingüístico. El primer resultado da cuenta de un concepto de evaluación que se define en coherencia a la identidad MAPUCE. 
Yo creo que estamos en una transición de cómo ir abordando las evaluaciones que comúnmente utilizamos... rúbricas, listas de cotejo, pero utilizando las conductas MAPUCE pero de todas maneras igual nosotros conocemos medianamente nuestra cultura, igual en todas las culturas creo yo que el accionar de los niños, jóvenes y personas en si es evaluado, nada pasa casualmente, nada se hace por hacer porque siempre alguien está observando y valida o invalida el accionar (...). Así que nosotros no tenemos una evaluación que digamos, aquí está, pero como docentes hemos tenido conversaciones de ¿cómo abordarlo? Pero no lo hemos definido todavía, aunque en lo cultural ya existe, como el KIMKANTUN (Profesor 1 MAPUCE, Extracto de Conversación dialógica).

La cita explica que la comunidad MAPUCE no tiene un concepto escolarizado de evaluación, pero que, sin embargo, culturalmente existe el KIMKANTUN como el proceso de acompañamiento a la enseñanza y aprendizaje de los niños, niñas y jóvenes. A este respecto, esta comunidad indígena busca resignificar la evaluación occidental y avanzar en un concepto coherente a su identidad.

En una línea similar, una profesora explica que la evaluación en contexto indígena debe ser un proceso cotidiano y lo explica en función del juego:

En cuanto al conocimiento MAPUCE, uno va evaluando según las etapas, por ejemplo: a los más pequeñitos, usted le habla nomás (en MAPUZUGUN) porque ellos escasamente hablan, solo imitan y actúan... eso se llama KIMGOLI, aprender en el juego. Cuando se cría a un niño MAPUCE y él ve que todos hacemos LLELLIPUN (ceremonia), él imita, entonces va jugando y aprendiendo (Profesora 2 MAPUCE, Extracto de Conversación dialógica).

El concepto de evaluación en contexto MAPUCE debe rescatar los saberes propios de su cultura y lengua, así este proceso es contextual y no limita al estudiante a una sola cultura, sino con sentido de ampliación epistémica. Así como esta comunidad indígena respeta la escolarización occidental, promueven la importancia de la incorporación de sus propios conocimientos. Por lo que, la evaluación debe orientar a un diálogo de saberes, debido que "en todo KIMVN, nuestra evaluación MAPUCE debería considerar cómo el niño va aprendiendo dentro del proceso, vamos observando si el niño aprendió o no aprendió" (Profesor 1 MAPUCE, Extracto de Conversación dialógica).

El segundo resultado de la dimensión transformadora da cuenta de la observación y conversación como estrategia metodológica de evaluación.

Observándolos, observándolos, les hacemos preguntas en nuestra lengua, miramos siempre los avances que tiene. Utilizamos la observación de sus dibujos, cuando yo explico algo luego los dejo trabajando solos y después veo sus dibujos, el niño que entendió hace una buena interpretación (Profesora 3 MAPUCE, Extracto de Conversación dialógica).

La observación, si bien es una estrategia occidental que tiene instrumento propio como la pauta de observación o la rúbrica (entre otros instrumentos de corte cualitativo), la comunidad MAPUCE la dirige desde su cosmovisión y en lengua propia, y la finalidad 
de ésta es tanto el acompañamiento como la observación de la interpretación de los saberes que realiza el estudiante. Para la escuela chilena, la observación se utiliza mayoritariamente para acompañar el aprendizaje como proceso formativo, haciendo énfasis en los aciertos y desaciertos de los y las estudiantes por medio de la oralidad. Sin embargo, la comunidad de investigación desarrolla este método desde su comprensión y pensamiento. Siendo metodológicamente la observación fundamental por parte del profesorado para orientar la atención de los estudiantes y para éstos como fuente de interpretación de la realidad.

Finalmente, se valida como transformador la propuesta de una escala numérica como instrumento de evaluación.

Creemos que es necesario tener un instrumento de evaluación, aunque no creo que con una escala numérica del 1 al 7, sino más bien, con conceptos MAPUCE. Porque la cultura MAPUCE siempre se está evaluando, si no hizo bien o si cumplió... si lo hacemos numérico utilizar una escala del 1 al 4 porque el 4 es un concepto clave o el 8 por la dualidad (Profesor 2 MAPUCE, Extracto de conversación dialógica).

Para la evaluación en contexto escolar chileno, las notas (calificación) se expresan del número 1 al 7. Siendo el 1 una baja nota y el 7 la máxima. A diferencia de la comunidad MAPUCE, que las mismas notas no tienen significado porque no tienen una base epistémica sociocultural. Para esta comunidad es importante la dualidad, debido que todo tiene su compañero y compañera, como el día y la noche, el mar y la tierra; por esto, tienen significado los números duales, y para ellos el 4 es el número perfecto, porque representa equilibrio y armonía. Por lo tanto, este número significaría para sus niños, niñas y jóvenes que han alcanzado el nivel máximo. O también, el número 8, representando la dualidad.

En este sentido, se observa la importancia del legado histórico espiritual de la cultura MAPUCE, que no ven reflejada en la escuela occidental y por esto, promueven una apertura epistemológica que resignifica la evaluación. Se afirma lo anterior cuando una profesora dice que:

Las escalas a utilizar también se deben definir (...) algo cultural tiene que ser una escala propia. Va a ser un desafío pero hay que trabajar (...) No debería ser del 1 al 7, si es que se va a calificar, debería ser bajo otro sentido como el 4 (Profesora 2 MAPUCE, Extracto de Conversación dialógica).

Es un desafío la construcción de un instrumento particular de la cultura MAPUCE que refleje el aprendizaje en términos occidentales, porque para ellos no existe la calificación, sino más bien, la valoración del aprendizaje.

\subsubsection{Resultados dimensión exclusora}

La comunidad de investigación categorizó como exclusor un concepto de evaluación que la define como proceso homogéneo. La evaluación se entiende como un método jerárquico que no contempla en los contenidos curriculares (conocimientos), saberes propios de las culturas originarias. Necesariamente la comunidad indígena se adecúa a los requerimientos escolares occidentales. 
Tratar de homogenizar es como meter un elefante en un embudo, el Estado dice que la educación es para todos y no es así, la educación y la evaluación no está contextualizada de forma pertinente, nos obligan a adecuar su realidad a la de acá, sólo porque el Estado lo exige (Profesora 3 MAPUCE, Extracto de Conversación dialógica).

No obstante, la comunidad MAPUCE, concibe y vive entre dos racionalidades, una objetiva que da cuenta de la realidad occidental, y otra subjetiva, que guarda sus prácticas ancestrales, su cultura y lengua. De esta manera, desarrollan en la escuela un diálogo entre estas racionalidades: por un lado, son conscientes de la normativa educacional chilena y de su importancia como medio para la incorporación a esta sociedad de sus niños, niñas y jóvenes, y, por otro lado, al mismo tiempo desarrollan en su LOF el tipo de educación propio a su cultura.

Nosotros tenemos dos tipos de evaluación aquí: la evaluación que el sistema chileno nos da y la hacemos tal como ellos nos dicen, apegándonos a la normativa a la que estamos vinculados entendiendo que ellos son lo que aportan los recursos y nosotros debemos responder. Nosotros pensamos que si el sistema quiere eso nosotros se lo damos, pero en cuanto a lo que nosotros esperamos de nuestra parte cultural, ahí nosotros nos manejamos en nuestro propio terreno (Profesora 2 MAPUCE, Extracto de Conversación dialógica).

Dado lo anterior, la evaluación debe responder y ser coherente a estas dos racionalidades, sin desmedro una de la otra, sino en su conjunto para la búsqueda de una meta común: la revitalización de la cultura indígena en la escuela.

En síntesis, el objetivo 2, Definir el concepto de evaluación de aprendizajes en contexto MAPUCE BAFKEHCE y su metodología, devela la propuesta de un concepto coherente a su identidad, entendiéndose como una evaluación que ayuda en la comprensión de la realidad indígena para delinear vías de acceso a dicha realidad, es decir, la evaluación se resignifica junto a la comunidad. A diferencia de la evaluación occidental, que la comunidad MAPUCE entiende como jerárquica.

En dicho sentido, la evaluación es un proceso de acompañamiento cotidiano de los aprendizajes y no busca solamente su medición, sino junto a lo anterior, la revitalización de su KIMVN por medio de prácticas propias a su cultura y prácticas educativas occidentales, promoviendo un desarrollo gnoseológico y consciente del KIMKANTVN. El concepto propuesto está en plena coherencia con los elementos orientadores revisados en los resultados del objetivo 1. La metodología de observación se corresponde a su cosmovisión, como el desarrollo de una escala numérica, como una rúbrica, con base en la dualidad. Esto no se contrapone a la evaluación desde su comprensión occidental, sino que la enriquece y permite una valoración de los conocimientos desde un contexto situado, que puede ser generalizable y replicable a otros contextos educativos. Es decir, que la evaluación tenga sentido para el estudiante.

Por otro lado, se rechaza la concepción homogénea de la evaluación, que entiende todos los contextos educativos como similares, al igual que a todo el estudiantado. Por lo mismo, la comunidad de investigación avanza en su propuesta de diálogo entre ambas culturas, la indígena y la chileno occidental. 


\section{DISCUSIÓN Y CONCLUSIONES}

Un punto relevante por discutir es la distinción entre evaluación puramente occidental y una evaluación puramente indígena. En este sentido, los resultados apuntan a una comprensión de principios orientadores (objetivo 1) que permiten establecer un diálogo entre ambas racionalidades, occidental-chilena e indígena. Los cinco principios orientadores, KIMKANTUN, MAPUZUGUN, KIMCE, KIMVN y LOF, generan la comprensión del espacio-tiempo-territorialidad y lengua como eje vertebrador de la evaluación para contexto indígena.

Lo anterior es relevante ya que la comunidad MAPUCE de esta investigación critica el PEIB instalado desde el Ministerio de Educación, por su posición asimilacionista con el indígena; sin embargo, la misma comunidad intenta establecer el vínculo entre ambas culturas. Los principios orientadores no promueven una evaluación puramente MAPUCE, sino una valoración sobre su cultura que permite generar una propuesta evaluativa coherente a su cosmovisión. De esta manera, la formación de sus estudiantes se centra que puedan movilizarse en ambas racionalidades. La evaluación en contexto indígena debe partir desde el contexto situado para luego dar cuenta de los requerimientos curriculares estatales.

La evaluación occidental no da cuenta, como ya se ha mencionado, de la pertinencia cultural, y menos lingüística, por esto se debe seguir avanzando junto a comunidades indígenas en propuestas orientadoras que permitan la comprensión de la particularidad y complejidad de la comunidad indígena.

Los principios orientadores dan cuenta de una evaluación que tenga sostenibilidad cultural y curricular en el tiempo de escolarización. Éstos no se contraponen al currículum nacional, sino por el contrario, enriquecen la formación de los niños, niñas y jóvenes en contexto indígena.

Lo anterior se relaciona con la naturaleza ética de la evaluación (Cram y Mertens, 2016) que estos principios orientadores nos enseñan que dicha naturaleza ética tiene relación con la diversidad presente en el contexto escolar, sobretodo cuando se trabaja junto a la comunidad indígena que significa no solo respetar su cultura y lengua, sino que comprender todos los fenómenos visibles e invisibles que su cosmovisión valora.

Los resultados del objetivo 1 se relacionan con las características propias de la comunidad MAPUCE respecto de la sociedad no-indígena en Chile, debido que la naturaleza de la realidad en evaluación, reconoce a la persona (CE) en una continua construcción de la identidad, es decir, "un proceso permanente de liberación que se desarrolla a partir del constante discernimiento al interior de cada grupo social y cultural con que se identifica cada individuo" (Quilaqueo y Quintriqueo, 2017, p. 31).

Los mismos resultados sostienen que cuando la evaluación se enfrenta a este contexto educativo indígena, epistémicamente, afirma la propia identidad cultural con relación a otras culturas, evitando el asimilacionismo. La evaluación promueve una percepción enriquecedora de la diferencia cultural, así personas indígenas y no-indígenas que comparten la escolaridad, suponen la aceptación de sus culturas para un enriquecimiento conjunto de todos y todas; solo así se posibilita un desarrollo evaluativo que no dificulte los avances y logros educativos, ya que no se trata de un proceso sectorial de las diferentes estructuras sociales. De esta manera el componente epistemológico en evaluación en contexto indígena promueve que el profesorado no-indígena que se enfrenta a contexto escolares con población indígena, debe tener una actitud comprensiva e ingenua, frente a las formas de conocimiento 
de la cultura indígena y debe enfrentarse sin respuestas predeterminadas a la realidad escolar, sino desde una conciencia histórica del sujeto (Mejía, 2014; Zemelman, 2005).

La relación entre el/la evaluador/a con la realidad evaluativa que desea conocer, va en estrecha relación con una episteme situada históricamente y que la comunidad indígena mantiene, desarrolla y decide qué, cómo y para qué dicho saber/conocimiento va a ser evaluado, cómo evaluarlo y para qué evaluarlo, porque las prácticas epistémicas son extendidas a distintas formas de construcción, según el territorio y la comunidad indígena que vive allí. Según su lengua, sus decisiones de potencialidad de desarrollo en sus niños, niñas y jóvenes. Los resultados del objetivo 1, desde el punto de vista de la epistemología en evaluación, dan cuenta de cómo el evaluador se enfrenta ingenuamente a una construcción de pensamiento y cómo es capaz de generar conocimiento evaluativo junto a la comunidad.

En este sentido, cobra relevancia la orientación que plantea Schmelkes (2015), cuando dice que la evaluación en contexto indígena debería ser en su lengua originaria. Esto porque el "mundo" se construye a partir de los componentes lingüísticos propios a la cultura de origen, como las relaciones entre el "ser" y el cosmos, con el territorio inmediato, con las demás personas de su comunidad y con el universo entero.

Los resultados del objetivo 2, básicamente metodológicos, sostienen que el método de evaluación en contexto indígena es construido con base al territorio donde se ubica la comunidad, rescatando metodologías tradicionales y apuntando a la generación de nuevas formas evaluativas. A este respecto, la comunidad mapuce es mayoritariamente una cultura oral, de transmisión de conocimiento por medio de narraciones, sueños, por lo que las instancias de evaluación deben ser coherentes a estas prácticas, por lo mismo, es la observación del proceso narrativo lo que la comunidad genera como método evaluativo.

La metodología de evaluación en contexto indígena, por un lado, recoge las prácticas de enseñanza propias de las culturas y por otro, junto a la comunidad desarrolla estrategias coherentes y apropiadas a su cosmovisión. Así, la metodología apunta a los conocimientos culturales y prácticas desarrolladas por los estudiantes, habilidades y niveles de comprensión adquiridos por estos haciendo énfasis en la capacidad para que puedan utilizarlos en otros contextos. Es relevante destacar que no existe un solo método o una guía (manual de evaluación), sino múltiples y variadas estrategias según las prácticas ancestrales y su diálogo con la realidad.

Lo anterior, motiva a la resignificación de la escala numérica con sentido sociohistórico y cultural. Es decir, que la calificación tenga un significado "más allá de una nota" para el estudiante. Eisner (1998) critica la concepción numérica o alfabética de la calificación, preguntando básicamente: ¿Qué significa para el estudiante un número? Por esto, el sentido cultural de la numeración en cuanto la dualidad y el número 4 como perfección, tiene para los estudiantes MAPUCE una connotación cosmogónica y no puramente instrumental.

Este estudio, con resultados preliminares de una investigación más grande como explicáramos en la introducción, pretende ayudar en la búsqueda y promoción de una evaluación con pertinencia cultural y lingüística a las comunidades indígenas en Chile.

\section{REFERENCIAS BIBLIOGRÁFICAS}

Acuña, M. E. (2012). Perfil de educadores tradicionales y profesores mentores en el marco de la implementación del sector de lengua indígena. Santiago: Unicef. 
Calfuqueo, J., Cubillos, F., Pinto, D. y Del Pino, M. (2018). La experiencia de construcción curricular en una escuela de la Araucanía y su análisis desde la justicia social. En D. Ferrada (Ed.), Políticas y su impacto en las comunidades. Investigación en educación para la justicia social (pp. 73-92). Talca: UCM.

Calvino, H. (2013). Across the colonial divide: Conversations about evaluation in indigenous contexts. American JournalofEvaluation, 34(3),339-355.doi:https://doi.org/10.1177/1098214013489338.

Cram, F. (2001). Rangahau Māori: Tona Tika, Tona Pono. En M. Tolich (Ed.), Research ethics in Aotearoa. Auckland, New Zealand (pp. 32-52). Auckland: Longman.

(2009). Maintaining indigenous voices. In D. Mertens, y P. Ginsberg (Eds.). SAGE Handbook of social science research ethics. Thousand Oaks, CA: Sage. doi: http://dx.doi. org/10.4135/9781483348971.n20.

Cram, F. \& Mertens, D. (2016). Negotiating solidarity between indigenous and transformative paradigms in evaluation. Evaluation Matters-He Take Tō Te Aromatawai, 2, 161-189. doi: http:// dx.doi.org/10.18296/em.0015. 2016.

Cubillos, F. (2016). Conocimiento territorial ancestral de las comunidades Mapuce Bafkehce del Aija Rewe Fvzv Bewfv Mapu Mew. 2016. Tesis (Doctorado). Universitat Autònoma de Barcelona. Departament de Didàctica de la Llengua, de la Literatura i de les Ciències Socials.

Del Pino, M., Pino, M. y Monsalva, G. (2018). Salud y sanación: Kvme mogen como ámbito del saber educativo mapuce. En D. Ferrada (Ed.), Reflexiones y experiencias educativas desde las comunidades. Investigación en educación para la justicia social. (pp. 19-37). Talca: UCM.

Drawson, A. S., Toombs, E. y Mushquash, C. J. (2017). Indigenous research methods: A systematic review. The International Indigenous Policy Journal, 8(2). doi: http://dx.doi.org/10.18584/ iipj.2017.8.2.5.

Eisner, E. (1998). Educar la visión artística. España: Paidós.

Fals Borda, O. (1979). El problema de cómo investigar la realidad para transformarla por la praxis. Colombia: Ediciones Tercer Mundo.

Fernández, M., Jofré, D., Mancini, G., Navarrete, D. y Venegas, G. (2012). Conocimiento consuetudinario de las comunidades MAPUCE BAFKEHCE DEL AIJA REWE LEUFU BUDI: una propuesta didáctica de educación sobre AZ MOGEN WAJONTU MAPU MEW cosmovisión tiempo-espacio. Tesis (Pregrado). Universidad Metropolitana de Ciencias de la Educación.

Ferrada, D. (2017). La investigación participativa dialógica. En R. Pantorra y R. Angulo (Ed.), Investigación o Metodología cualitativa en educación (pp. 177-189 o 187-201). Madrid: Miño y Dávila.

Ferrada, D. y Del Pino, M. (2018). Dialogic-kishu kimkelay ta che educational research: participatory action research. Revista: Educational action research, 26(4), 533-549. doi: https://doi.org/10.1 080/09650792.2017.1379422.

Ferrada, D., Villena, A., Catriquir, D., Turra, O., Pozo, G., Schilling, C. y Del Pino, M. (2014). Investigación dialógica-kishu kimkelay ta che en educación. Revista: REXE, 13(26), 33-50.

Heron, J. y Reason, P. (1997). A participatory inquiry paradigm. Revista Qualitative Inquiry, 3(3), 274-294.

Kawakami, A., Aton, K., Cram, F., Lai, M. y Porima, L. (2007). Improving the practice of evaluation through indigenous values and methods: Decolonizing evaluation. Practice-returning the gaze from hawaï̈i and aotearoa. Hülili: Multidisciplinary research on hawaiian well-being, 4(1), 319-348.

King, L. y Schielmann, S. (2004). El reto de la educación indígena: experiencias y perspectivas. Unesco.

Lagos, C. (2015). El programa de educación bilingüe y sus resultados: ¿perpetuando la discriminación? Revista Pensamiento educativo, 52(1), 84-94. doi: http://dx.doi.org/10.7764/PEL.52.1.2015.7.

Mejía, M. R. J. (2014). La educación popular: una construcción colectiva desde el Sur y desde abajo. Revista: Arquivos analíticos de políticas educativas, 22(62), 227-249. doi: http://dx.doi. 
org/10.14507/epaa.v22n62.2014.

Mertens, D. M. (2013). Social transformation and evaluation. In M. Alkin (Ed.), Evaluation roots (pp. 229-240). Los Ángeles: University of California.

Ministerio de Educación (1996). Decreto 40 Objetivos fundamentales y contenidos mínimos obligatorios de la educación básica y media. Santiago: MINEDUC.

. (2009). Decreto 280 Objetivos fundamentales y contenidos mínimos obligatorios de la educación básica y media. Santiago: MINEDUC.

Ministerio de Planificación y Cooperación. (1993). Ley N ${ }^{\circ} 19.253$ Establece normas sobre protección, fomento y desarrollo de los indígenas, y crea la Corporación Nacional de Desarrollo Indígena. Santiago: Gobierno de Chile.

Naciones Unidas. (2007). Declaración de las Naciones Unidas sobre los Derechos de los Pueblos Indígenas. Última visita 15 de mayo de 2018. Recuperado de: https://www.un.org/esa/socdev/ unpfii/documents/DRIPS_es.pdf

OIT. (1989). Convenio 169 sobre Pueblos Indígenas y Tribales. Última visita 20 de mayo de 2018. Recuperado de: https://www.ilo.org/dyn/normlex/es/f?p=NORMLEXPUB:12100:0::NO: :P12100_INSTRUMENT_ID:312314.

Pinto, R. (2013). Construyendo currículum emergente en Llaguepulli. Revista REXE, 12(4), 15-36.

Quilaqueo, D. y Quintriqueo, S. (2017). Métodos educativos mapuches: retos de la doble racionalidad educativa. Aportes para un enfoque educativo intercultural. Temuco: Ediciones Universidad Católica de Temuco.

Rivera, D. y Fuentes, V. (2017). Propuesta evaluativa para la escuela KOM PU LOF ÑI KIMELTUWE en los subsectores: AZ MOGEN WAJONTU MAPU MEW o cosmovisión tiempo-espacio y AMULEPE TAIÑ MOGEN o historia y cultura MAPUCE. Tesis (Pregrado). Universidad Metropolitana de Ciencias de la Educación.

Schmelkes, S. (2015). Hacia una evaluación con enfoque intercultural. Pensamiento pedagógico, Tarea, 89, 60-64.

Williamson, G., y Flores, F. (2015). Estado del arte de la Educación Intercultural bilingüe en Chile, 1990-2013. Teтисо, ICIIS y Ediciones Universidad de La Frontera.

Yopo, M. (2012). Políticas sociales y pueblos indígenas en Chile: Aproximación crítica desde la noción de agencia. Universum (Talca), 27(2), 187-208. doi: https://dx.doi.org/10.4067/S071823762012000200011.

Zemelman, H. (2005). Voluntad de conocer. El sujeto y su pensamiento en el paradigma crítico. Barcelona: Anthropos. 
